\title{
Aeroelastic Wingbox Stiffener Topology Optimization
}

\author{
Bret K. Stanford* \\ NASA Langley Research Center, Hampton, VA, 23681
}

\begin{abstract}
This work considers an aeroelastic wingbox model seeded with run-out blade stiffeners along the skins. Topology optimization is conducted within the shell webs of the stiffeners, in order to add cutouts and holes for mass reduction. This optimization is done with a global-local approach in order to moderate the computational cost: aeroelastic loads are computed at the wing-level, but the topology and sizing optimization is conducted at the panel-level. Each panel is optimized separately under stress, buckling, and adjacency constraints, and periodically reassembled to update the trimmed aeroelastic loads. The resulting topology is baselined against a design with standard full-depth solid stiffener blades, and found to weigh $7.43 \%$ less.
\end{abstract}

\section{Introduction}

Most examples of aeroelastic wingbox tailoring optimization in the literature involve identifying the best stiffness and inertial properties of the various shell members that comprise the structure: ribs, spars, stiffeners, and (most predominately) skins. Far less common are examples of aeroelastic topology optimization involving the optimal layout of material within the wingbox. These problems are typically more difficult to solve, and can take three different forms. First, the optimal layout of the web members (ribs, spars, stiffeners) may be located, considering various spacings, orientations, curvilinearity, and connectivity. ${ }^{1,2}$ Second, a series of lightening holes may be introduced and then optimized within these web members, for further weight reductions. ${ }^{3-5}$ Third, rib/spar/stiffener layouts may be abandoned altogether, in favor of some more general distribution of three-dimensional material. ${ }^{6,7}$

For all three versions of wingbox topology optimization, skin buckling during maneuver loads is the key physical design metric of interest. Particularly for the high aspect ratio transport wings considered here, the skin stiffness properties dominate the global aeroelastic response, and the layout details of the internal members are less important to the global behavior. ${ }^{8}$ However, the way in which the internal wingbox layout reinforces those skins will dictate their buckling resistance. Skin stiffness will also play a role in buckling computations, and so topology optimization should be considered concurrent with skin sizing.

Skin buckling is a difficult metric to accommodate, particularly in topology optimization. As the nature of the internal layout evolves during the design process, the identity of the critical buckling mode will likely change, shifting to a different location along the wing skin, and perhaps changing from a global to a local mechanism (or vice-versa) as well. In order to maintain a smooth design space for gradient-based topology optimization, subcritical buckling modes must be computed at each design iteration, so the optimizer can "see them coming", in the event that they may become critical at the next iteration. ${ }^{9}$ For a realisticallypaneled wingbox, hundreds of computationally-expensive eigenmodes could reasonably play a role during topology optimization, with aggressive switching of the critical mode from one iteration to the next.

The current work utilizes the second type of topology optimization described above, and attempts to optimize lightening holes within the skin stiffeners. The number and orientation of the run-out stiffeners are fixed, as are the topological details of each rib, which are not optimized here. In order to ease the burden of computing and organizing buckling eigen-data, a global-local approach is used. Trimmed aeroelastic maneuver loads are computed for the entire wing, where stiffeners are modeled implicitly (smeared). ${ }^{10}$ Deformations are then interpolated onto a local skin panel mesh (where a panel is bordered by two ribs and two spar segments), where the blade stiffeners are modeled explicitly.

* Research Aerospace Engineer, Aeroelasticity Branch, bret.k.stanford@nasa.gov, AIAA Senior Member. 
The panel thickness and stiffener topology are then optimized for minimum mass, under stress and buckling constraints, where buckling is considered to be confined to that single panel. Upon convergence (or alternatively, after only a few iterations), the process is repeated with the next panel along the wing; although ideally, multiple panels can be optimized in parallel. Once every panel has been optimized, the wing is reassembled, stiffeners are resmeared, and a new set of trimmed aeroelastic maneuver loads are computed at the wing-level. The panel-by-panel topology optimization process then restarts. The entire outer-loop is repeated until the global-level wing mass converges. Such a global-local wingbox design decomposition is a well-known strategy; ${ }^{11-13}$ the added level of complexity here is the use of topology optimization at the local panel-level.

\section{Problem Setup}

All of the work in this paper is conducted on the conceptual Common Research Model (CRM). The $1 g$ outer mold line for the CRM is described in Ref. 14, and a jig shape CRM wingbox was subsequently developed by Kenway et al. ${ }^{15}$ The CRM jig shape is used in this work, and can be seen in Fig. 1. This transonic transport has a wing span of $58.7 \mathrm{~m}$, a mean aerodynamic chord of $7.0 \mathrm{~m}$, a taper ratio of 0.25 , a sweep angle of $35^{\circ}$, and a cruise Mach number of 0.85 . The topology of the wingbox in Fig. 1 consists of 43 ribs, leading and trailing spars, and upper and lower surface skins. All shell members are outfitted with T-shaped stiffeners, where the flange is bonded to the shell members. The skin stiffener pitch is 30.0 $\mathrm{cm}$, the spar stiffener pitch is $15.0 \mathrm{~cm}$, and the rib stiffener pitch is $22.5 \mathrm{~cm}$. At the global wing-level, the stiffeners are not modeled explicitly, but instead smeared into the shell stiffness properties. ${ }^{10}$ At the local skin panel-level, stiffeners are modeled explicitly, as seen on the right of Fig. 1.
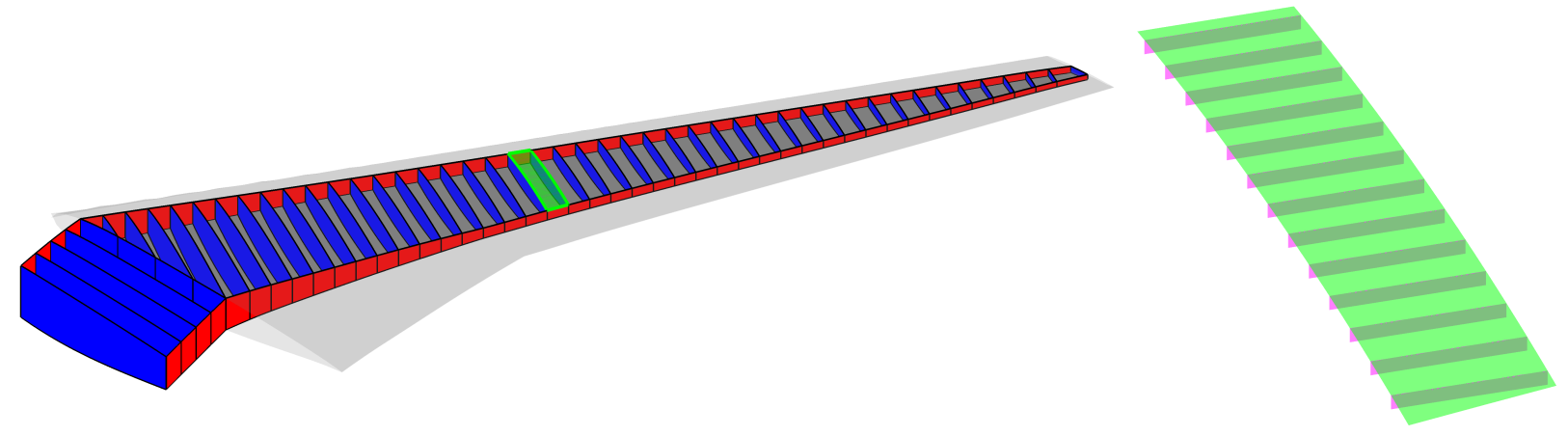

Figure 1. Wingbox and outer mold line of the CRM, as well as a representative stiffened panel.

Aeroelastic maneuver loads are computed by coupling a linear shell finite element model of the wingbox in Fig. 1 to a linear aerodynamic panel tool, whose mesh is seen on the left of Fig. 2. Lumped mass representations of the fuel, engine, and leading/trailing edge control surfaces are also seen in Fig. 2. Two maneuvers are considered in this work: a $2.5 g$ pull-up, and a $-1 g$ push-over, both at sea level and Mach 0.64 , and with full fuel. The angle of attack and the tail elevator are used to trim the aeroelastic system. For a half-vehicle, fuel mass is set to $62,000 \mathrm{~kg}$, engine mass to $11,400 \mathrm{~kg}$, and leading/trailing edge control surface mass to $9,400 \mathrm{~kg}$. Unmodeled mass for the half-vehicle is set to $53,000 \mathrm{~kg}$. Structural wingbox mass, including a $25 \%$ knock-up for secondary mass, will vary during optimization between 10,000 and 15,000 kg. Given these values, the take-off gross weight (TOGW) for the entire vehicle is roughly $300,000 \mathrm{~kg}$.

Having computed the wing-level aeroelastic deformations for each maneuver load case, these deformations are interpolated onto the boundary of a selected panel mesh, whose mesh density is finer than what is used at the wing-level. Interpolation is computed via the shape functions of the wing-level shell finite elements. Having computed these edge motions, the interior deformations of the panel model are computed as:

$$
\boldsymbol{K}_{11} \cdot \boldsymbol{u}_{1}=-\boldsymbol{K}_{12} \cdot \boldsymbol{T} \cdot \boldsymbol{u}_{\text {wing }}
$$

where $\boldsymbol{K}$ is the panel stiffness matrix, the 1 subscript represents interior panel deformations, the 2 subscript represents panel edge degree of freedoms, $\boldsymbol{T}$ is the wing-panel interpolation matrix, and $\boldsymbol{u}_{\text {wing }}$ is the winglevel panel deformation. The panel stiffness matrix is computed using the well-known Solid Isotropic Material 


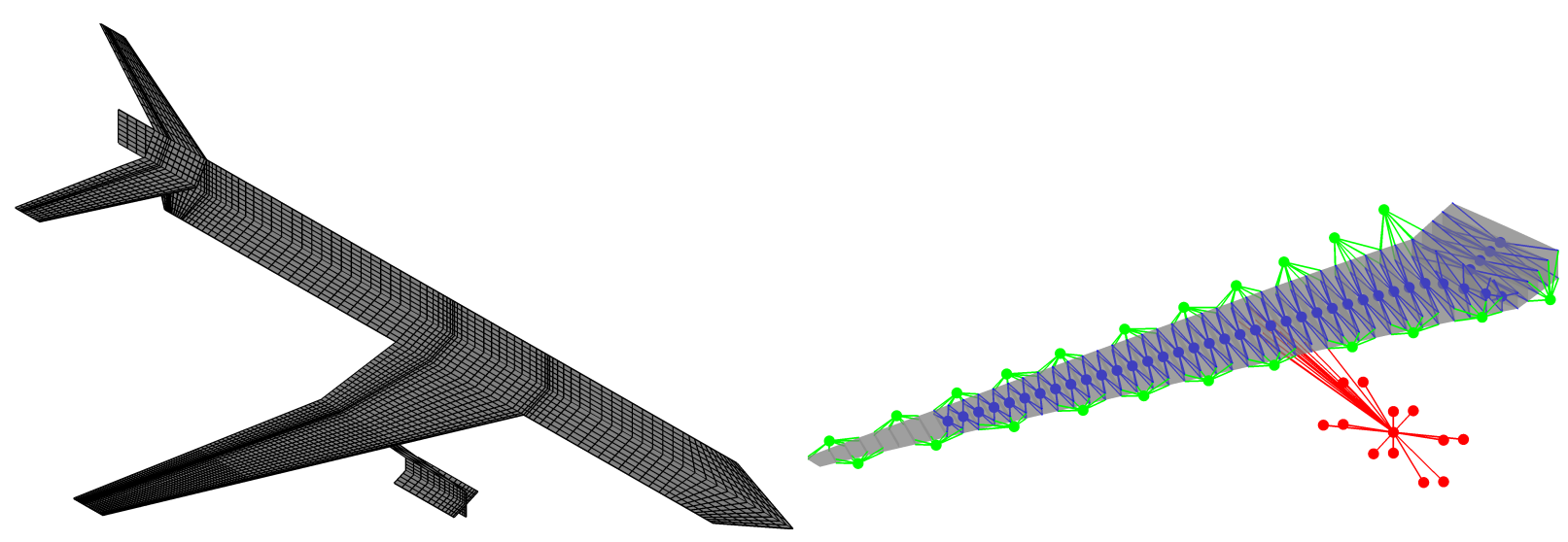

Figure 2. Aerodynamic paneling (left) and lumped mass representations (right).

with Penalization (SIMP) method: ${ }^{16}$

$$
\boldsymbol{K}=\sum_{e}\left(\boldsymbol{K}_{m, e} \cdot t_{e}+\boldsymbol{K}_{b, e} \cdot t_{e}^{3}\right) \cdot x_{e}^{p}
$$

The panel stiffness matrix is assembled over each shell element $e$, where $\boldsymbol{K}_{m, e}$ and $\boldsymbol{K}_{b, e}$ are the membrane and bending stiffness matrices (for a unit thickness), $t_{e}$ is the element thickness, and $x_{e}$ is the element topology design variable. The power $p$ is typically set to 3 for the SIMP method.

Within the stiffeners, the topology variables $x_{e}$ may vary from 1 (solid) to some very small nonzero value (void). Within the skin panel, these variables are fixed at 1, since topology optimization is only conducted within the stiffeners. Having assembled the panel stiffness matrix (Eq. 2), and computed the panel deformation (Eq. 1), the panel stresses are computed, as well as buckling factors via an eigen-equation. For buckling, stresses within the stiffeners are assumed to be zero, thereby "forcing" the eigemodes into the skin. The first (most critical) $N$ buckling modes are computed, where more modes are computed for panels with more stiffeners (such as found near the wing root).

The panel-level topology optimization problem is written as:

$$
\begin{gathered}
\text { minimize } \\
\boldsymbol{x}, t, t_{s}
\end{gathered} \quad \text { mass } \quad \text { s.t. }: \begin{cases}K S_{\sigma, i} \leq 1 & i=1, \ldots, N_{L} \\
K S_{\mu, i} \leq 1 & i=1, \ldots, N_{L} \\
\left|t-t_{s}\right| \leq \delta & \\
\left|t-t^{\text {neighbor }}\right| \leq \delta_{t} & \\
\left|t_{s}-t_{s}^{\text {neighbor }}\right| \leq \delta_{t_{s}} & \end{cases}
$$

The objective function is structural mass; the skin thickness $t$, stiffener thickness $t_{s}$, and vector of topology variables $\boldsymbol{x}$ are used as design variables. For this work, $t$ is considered uniform over a skin panel and $t_{s}$ is uniform over a set of panel stiffeners, though the topology obviously varies within those stiffeners, as dictated by $\boldsymbol{x}$. The Kreisselmeier-Steinhauser method ${ }^{17}$ is used for constraint aggregation. The von Mises stress for each skin panel shell element is aggregated into a single $K S_{\sigma}$ function for each load case, and forced to be less than one. A similar aggregation is performed for each of the $N$ computed buckling factors, for a given load case $\left(K S_{\mu}\right)$. Both stress and buckling constraints contain a safety factor of 1.5 .

Finally, a set of adjacency constraints are enforced, dictated by eventual manufacturing concerns. The first ensures against a large discrepancy between panel and stiffener thickness, forcing the difference to be less than $\delta$. The second forces the panel thickness to be within $\delta_{t}$ of the thickness of the neighboring (outboard) panel, and a similar neighbor constraint is enforced for stiffener thickness $\delta_{t_{s}}$. This optimization problem is solved using the Method of Moving Asymptotes (MMA). ${ }^{18}$ This problem can be driven all the way to convergence, or more likely, only a limited number of iterations can be performed.

This panel-level topology optimization process can be repeated for each of the skin panels: 48 panels along the upper skin, and 48 panels along the lower skin. Ideally, this process is done in parallel, as the topology optimization process for a given panel is assumed to be unaffected by the adjacent panels. Upon 
completion of this panel-by-panel process, the stiffeners in each panel are resmeared, and the complete wingbox is reassembled. For this work, the smeared stiffener height is assumed to be uniform over a given panel: in reality, there will be topological variations in stiffener properties throughout the panel. This uniform smeared stiffener height is simply computed such that the smeared stiffener mass within a panel (at the wing-level) is equal to the topologically-detailed stiffener mass (at the panel-level).

Having reassembled the wingbox, aeroelastic maneuver loads are recomputed at the wing-level, and the panel-by-panel topology optimization repeats, driven by the new wing deformation vector $\boldsymbol{u}_{\text {wing }}$. Panel-level design variables are copied from the final result of the previous topology optimization loop. The entire process repeats until the change in total wingbox mass between consecutive outer-loop iterations is below some tolerance.

As noted above, each panel is optimized in parallel, though there are actually intrapanel dependencies that will impact the aggregate design process. These dependencies are most explicitly true for the neighbor adjacency constraints, where the thickness of a panel must be within $\delta_{t}$ of its neighbor's thickness. In reality the neighboring panel thickness is a moving target (since this panel is being optimized concurrently), but this is idealized here by freezing $t^{\text {neighbor }}$ in Eq. 3 for a given panel topology optimization. This mismatch is reconciled at the end of the topology optimization process, when the wing is reassembled with updated sizing variables, global aeroelastic loads are recomputed, and an additional round of topology optimization commences. Similar issues arise from the simple fact that changing the stiffness properties of a given panel will impact the loads of its neighboring panel, which is again ignored at the local topology optimization level (but as above, reconciled when the wing is reassembled).

The number of outer-loop (wing-level) design iterations can likely be decreased by optimizing panels in serial, allowing changes in already-optimized panels to be immediately accounted for while optimizing the current panel. The current method of optimizing panels in parallel will likely have a much lower wall-clock time, however, given the substantial cost reduction afforded by parallel computing.

\section{Results}

The baseline distribution of shell thickness, smeared stiffener thickness, and smeared stiffener height is seen in Fig. 3. Shell thickness and stiffener thickness values are taken from Ref. 15, and the smeared stiffener height (in the skins) is assumed to be $80 \mathrm{~mm}$ throughout. A uniform baseline topology value of $x_{e}=0.5$ throughout every stiffener is used as well, which decreases the effective smeared stiffener height to $40 \mathrm{~mm}$, as discussed above. Rib and spar web sizing properties are not optimized in this work, and are fixed to the values seen in Fig. 3 throughout. The $2.5 \mathrm{~g}$ wing deformations for this case are shown in Fig. 4, along with von Mises stresses (normalized by the yield stress) at both the wing-level and for a sample panel. Panel-based stresses show localized stiffener-based stress concentrations that are neglected at the wing-level, owing to the smearing approximation.

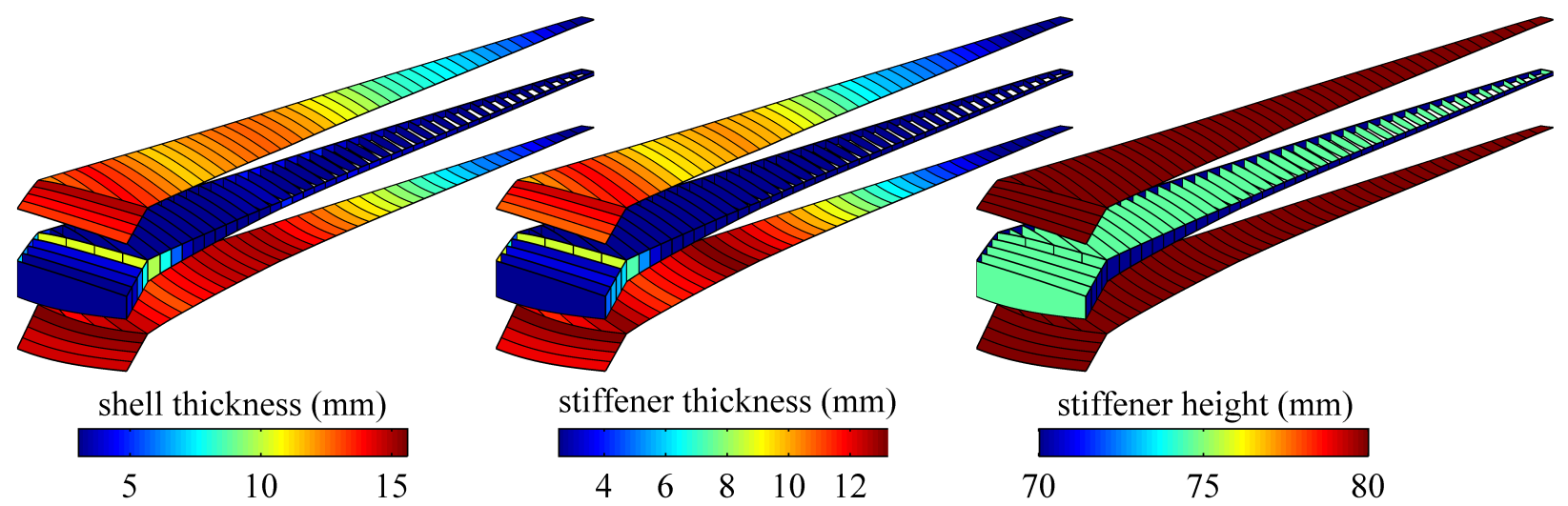

Figure 3. Baseline wingbox structural characteristics.

Next, topology optimization results are presented, using this baseline configuration as a starting point. The adjacency constraint boundaries are all set to $2.5 \mathrm{~mm}$ for $\delta, \delta_{t}$, and $\delta_{t_{s}}$ in Eq. 3, which is exactly satisfied by the baseline design in Fig. 3. Convergence results are shown in Fig. 5, in terms of three metrics. First, the 


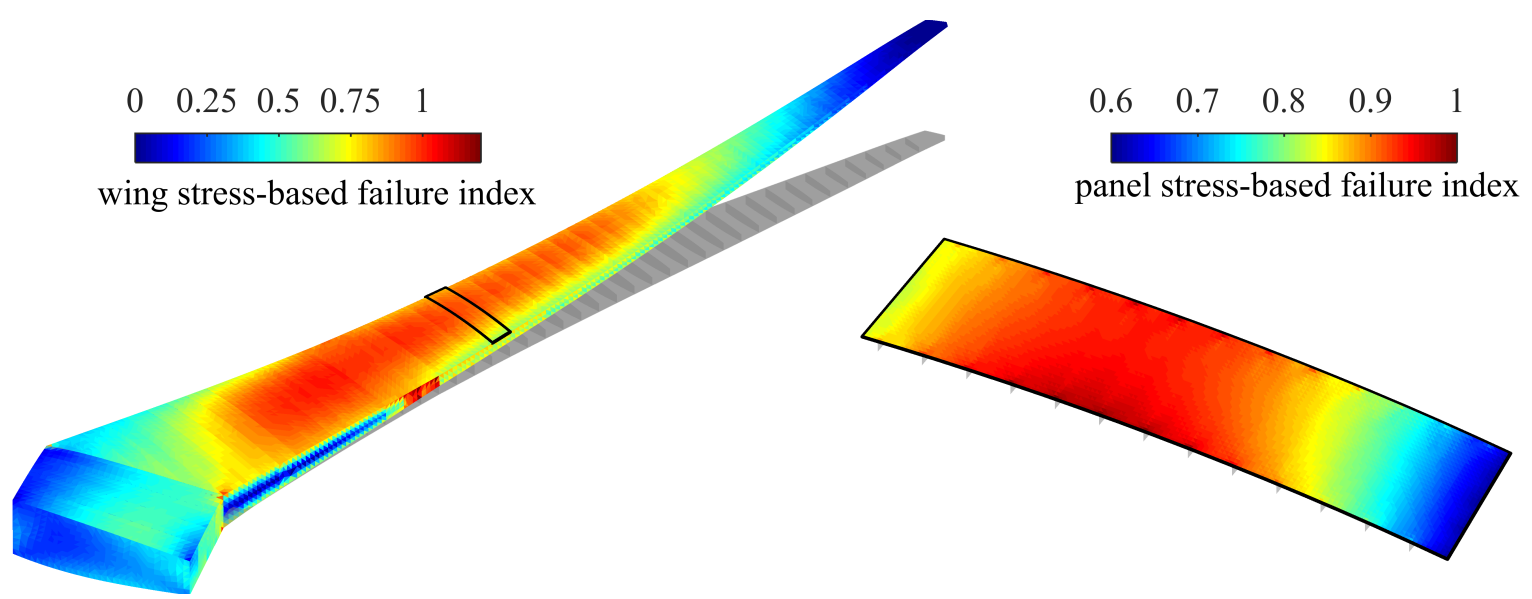

Figure 4. Wing-level and panel-level structural deformations for the baseline case.

structural wingbox mass is shown, which is the sum of the structural panel masses (optimized in Eq. 3) and the rib/spar masses (unoptimized, and held fixed throughout). Second, the convergence of this structural mass is shown. Finally, a grey metric for each panel is given by:

$$
\text { grey }=\boldsymbol{x}^{T} \cdot(1-\boldsymbol{x}) \cdot 4 / N_{x}
$$

This equation quantifies the topological convergence: if each $x_{e}$ variable has converged to the extremes of 1 (solid) or 0 (void), then grey will equal 0 . The upper bound of grey is 1 , found if every topology variable is equal to 0.5 . The average grey metric across all skin panels is the final quantity shown in Fig. 5, which starts at 1 for the baseline design, because each $x_{e}$ design variable has been initialized to 0.5 , as noted above. A cone-shaped spatial filter on topology design variables is used here, as is common in topology optimization. ${ }^{16}$ The filter radius within the stiffener webs is $10 \mathrm{~mm}$, compared to a stiffener height of $80 \mathrm{~mm}$ and a stiffener pitch of $30 \mathrm{~cm}$. The filter forces the optimizer to utilize intermediate $x_{e}$ values along the boundaries between solid and void, and so a grey metric of 0 will never be obtained by the optimizer.

The optimization in Fig. 5 is broken into 3 stages. The first stage is between outer-loop iterations of 1 and 16, with 10 inner-loop iterations each (i.e., 10 iterations of Eq. 3, for all 96 panels). The total structural wing mass during this stage drops from $14,762 \mathrm{~kg}$ (baseline) to roughly 13,450 kg. The macro-level structural details of the design are largely converged at this point, but the local topological features within many of the stiffeners are not, evinced by both the average grey metric (which drops from 1 to only 0.33 by iteration 16), and also the small undulations in the wing mass beyond iteration 12.

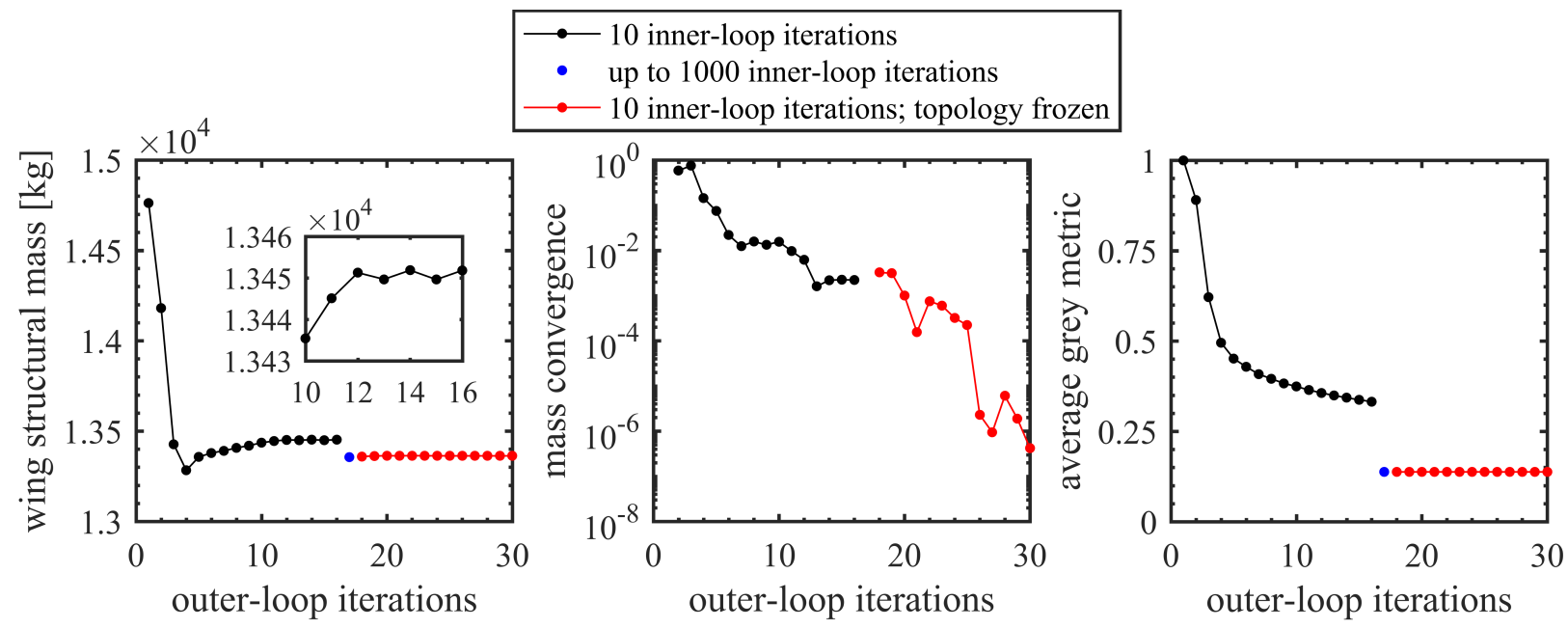

Figure 5. Outer-loop wing-level convergence histories. 
The average grey metric in Fig. 5 is continuing to drop at iteration 16, but very slowly. Panels with well-defined load paths (such as panels near the peak stress areas seen in Fig. 4, particularly in the upper skin where the $2.5 \mathrm{~g}$ load case buckling mechanisms are very aggressive) are topologically-converged by this point, but others are still forming. Rather than continue this costly process, iteration 17 attempts to topologicallyconverge each panel, by solving Eq. 3 for each panel until the local grey metric drops below 0.1 or through 1000 contiguous local iterations (whichever comes first). Once the wing is reassembled after this process, the average grey metric for the entire wing has dropped to 0.13 . The third and final phase of Fig. 5 is during outer-loop iterations above 17: here the topology design variables $\boldsymbol{x}$ are frozen, and only the $t$ and $t_{s}$ sizing variables are optimized, again using 10 inner-loop iterations at each outer iteration. The structural mass strongly converges to a value of 13,362 $\mathrm{kg}$ during this phase, with a difference between consecutive iterations on the order of $10^{-6}$.

Optimization details of a sample panel (the same panel shown in Fig. 1 and Fig. 4) are shown in Fig. 6, in terms of panel mass and the stress failure metric $K S_{\sigma}$ for the $2.5 \mathrm{~g}$ load case. The baseline panel is 122.0 $\mathrm{kg}$, and infeasible in terms of the stress constraint. The optimizer is able to satisfy the stress constraint at the end of the first 10 inner-loop iterations but when the wing is reassembled, and the aeroelastic loads recomputed, the constraint is again infeasible at the start of the next round of inner-loop panel design. This behavior, as discussed above, is due to intrapanel dependencies, which are ignored at the local panel design level, and also causes the undulations in the panel mass (objective function) in Fig. 6. The final panel mass is $127.9 \mathrm{~kg}$, where the skin thickness $(t)$ has increased from $11.89 \mathrm{~mm}$ to $14.12 \mathrm{~mm}$, and stiffener thickness $\left(t_{s}\right)$ from $9.39 \mathrm{~mm}$ to $11.62 \mathrm{~mm}$.

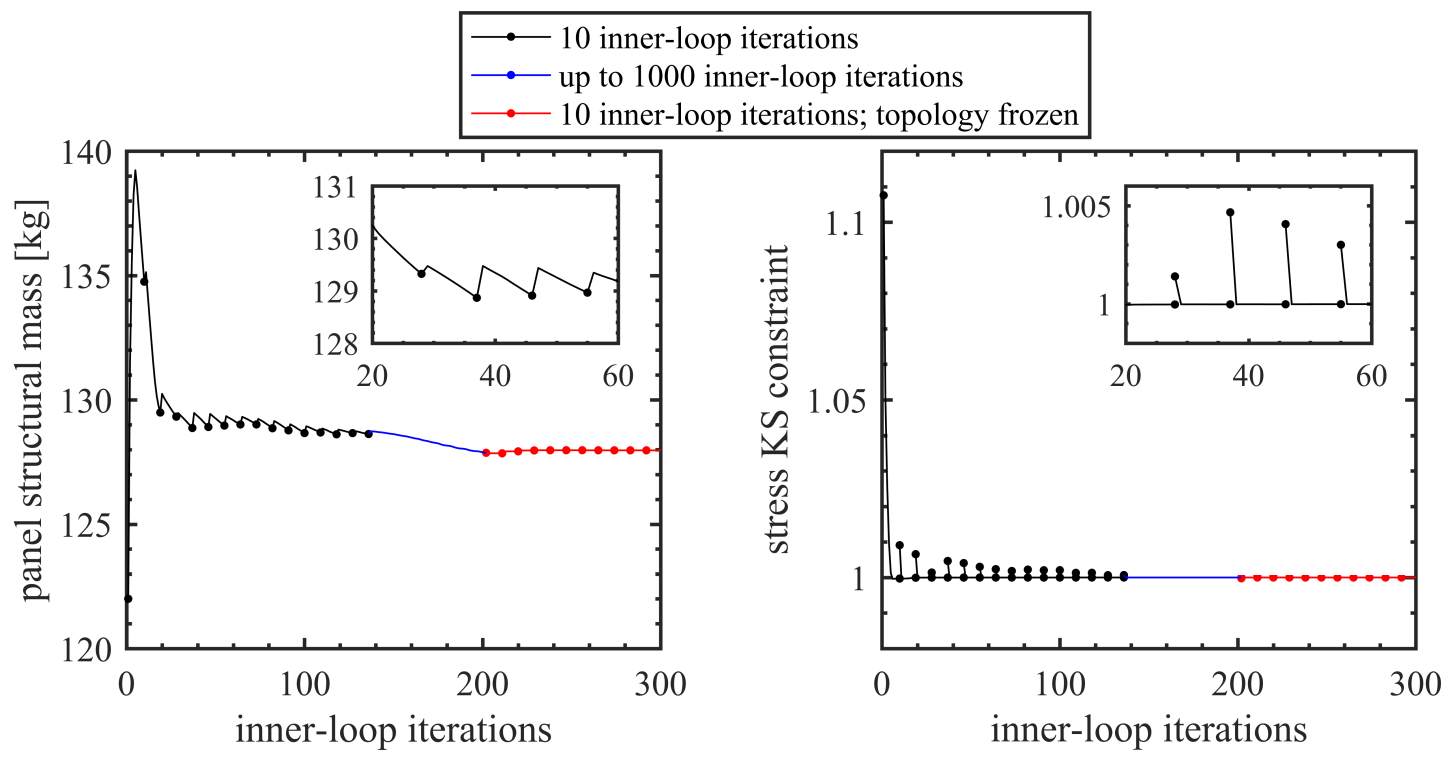

Figure 6. Sample panel-level convergence histories.

The critical buckling modes for this sample panel, before and after optimization, are seen in Fig. 7. Prior to optimization, the critical eigenvalue is above 1 , and so this constraint is violated. After optimization, the critical buckling eigenvalue is less than 1 , owing to the conservatism of $K S_{\mu} \cdot{ }^{17}$ It can also been seen that only the 5th buckling mode's identity has changed during the optimization process: the character of the least-stable eigenvector is largely the same. Had the baseline topology used here been stiffer (i.e., $x_{e}$ values larger than 0.5), the baseline buckling mode shape would likely have a localized character, with deformation confined between two stiffeners. In this notional case, the optimizer would have had to contend with strong changes in the identity of the critical buckling mode (as opposed to the optimal buckled shape in Fig. 7, which is a largely global shape), but the KS-based buckling constraint handling used in this work should be capable of accommodating such aggressive mode switching in a smooth manner.

The final topological layout for the wing is shown in the next several figures (Figs. 8 - 12) for groupings of panels along the upper and lower skins, near the wing root, midspan, and wing tip. It is again noted that the stiffener topologies are optimized along the entire wing (upper and lower skins), but topological details are too fine to be usefully plotted in a single figure: therefore, a piecemeal approach is taken here for 


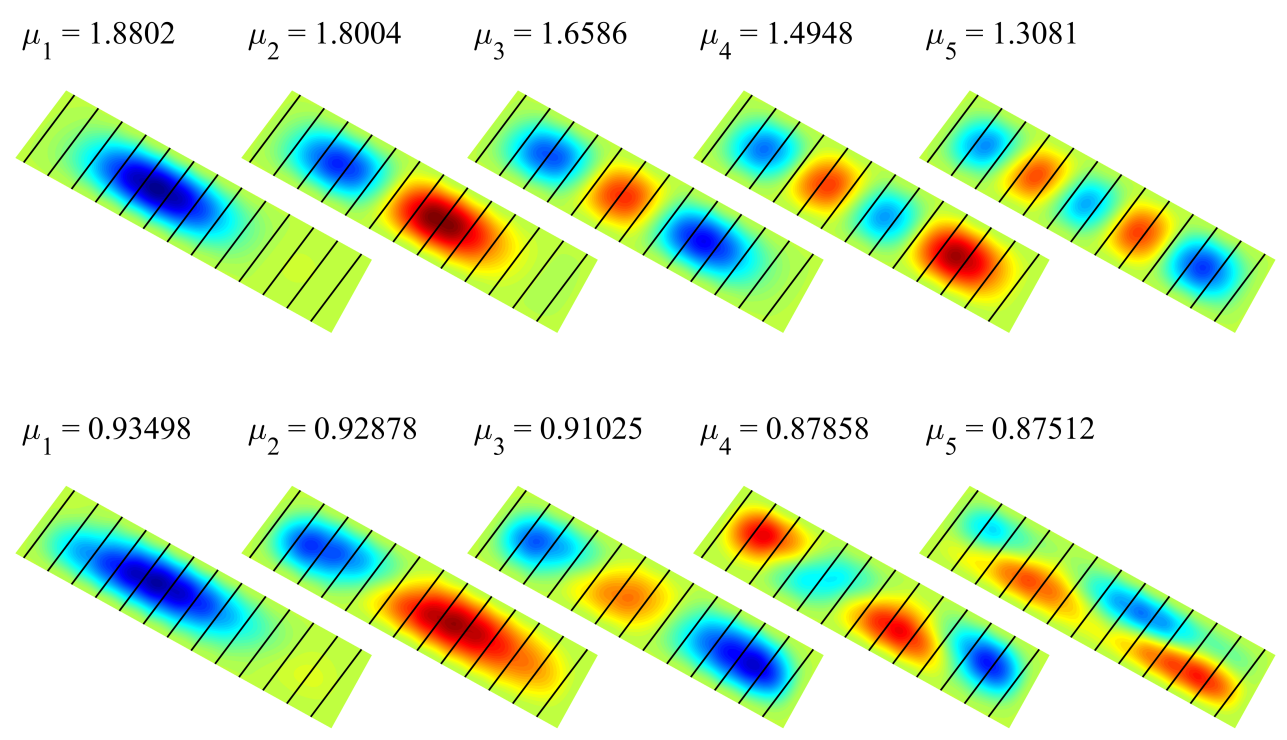

Figure 7. Critical buckling modes and eigenvalues before (top row) and after (bottom row) optimization.

plotting. Several conclusions may be drawn from these results. First, many (but not all) of the topologies within a given stiffener section (spanned between two ribs) may be characterized as a trapezoidal truss, with the long edge attached to the wing skin, the short edge laying along the bottom edge of the stiffener, and triangularly-oriented members in between. The optimizer consistently allocates material at the bottom stiffener edges for obvious reasons (increased bending moment of inertia for higher bending stiffness), but the inclusion of buckling or crippling ${ }^{8}$ constraints within the stiffeners themselves (which is not done here) will likely force the optimizer to temper such topological features.

It is also noted in Figs. 8 - 12 that there is very little topological communication within the stiffeners, between adjacent rib bays. In other words, there is little material allocated along the rib-stiffener boundaries. This material distribution is partly due to the fact that each panel is optimized independently (within the inner-loop iterations, at least), but mostly due to the fact that skin buckling deformation is largely confined toward the centers of each panel (as seen in Fig. 7 for example), and so stiffener topology is most effective when allocated in this area. This type of buckling eigenvector in turn is due to the partition of degrees of freedom in Eq. 1, which effectively renders the boundary conditions for the buckling problem as simplysupported along the panel edges. This assumption is reasonable if the ribs are relatively stiff, ${ }^{10,19}$ but thinner ribs would lead to buckling modes that span across multiple panels, and may in turn force the optimizer to allocate topology along rib-stiffener boundaries. Of course, such a global buckling mode would invalidate the core feature of this work, wherein panels are optimized individually and in parallel.

Final observations noted from Figs. 8 - 12 include the fact that far less material is allocated into the lower skin stiffeners compared to the upper skins, with some lower skin panels (not shown in the figures) completely bereft of any stiffener material. This is due to the load case definition, where upper skin buckling is driven by the $2.5 \mathrm{~g}$ maneuver, but lower skin buckling driven by the less-aggressive $-1 \mathrm{~g}$ load case. Some regions of poor topological convergence (also discussed within the context of Fig. 5 via the grey metric) are seen as well, particularly near the wing root (Fig. 8) and the wing tip (Fig. 12). Various options (none exercised here) are available to force a clearer topology in these areas, including continuation methods ${ }^{16}$ or simply allowing for more than 1000 inner-loop iterations during outer-loop iteration 17 in Fig. 5.

The optimizer in Fig. 5 is able to decrease the structural weight from $14,762 \mathrm{~kg}$ to $13,362 \mathrm{~kg}$, but the initial design in this case is not a logical baseline for topological comparisons. The initial design has $x_{e}$ set uniformly to 0.5 , which is a logical starting point for SIMP-based topology optimization, but is not a manufacturable design. A better option is to compare the topologies in Figs. 8 - 12 to a case where the stiffener topologies are forced to be full-depth. The optimizer can choose how deep the stiffeners are, but cannot add lightening holes within those shell members. In addition to being manufacturable, such a design is also representative of conventional stiffened wing panels. ${ }^{8,10}$

The full-depth parameterization is summarized in Fig. 13, for graphical comparison with an optimal 


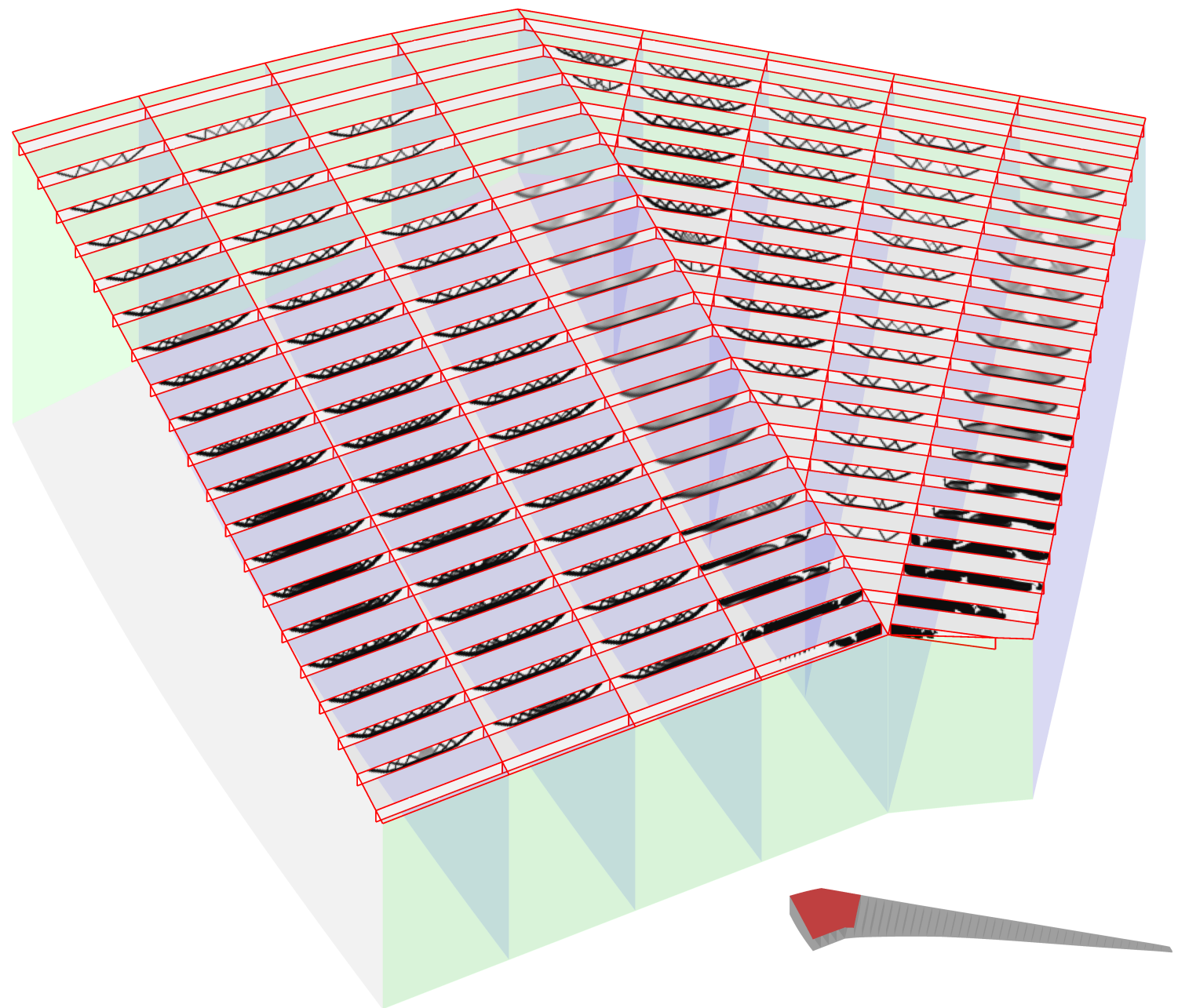

Figure 8. Upper skin topology near wing root.

stiffener topology taken from the previous result. There is a single topological design variable per panel, which governs the depth of the stiffeners. Shell elements above this depth line are assigned an $x_{e}$ of one, anything below the line is given an $x_{e}$ of zero, and there is a localized blending function between zero and one, to facilitate the topological sensitivity analysis. In this way, the method resembles level set techniques. ${ }^{20}$ The depth/height of each stiffener is spatially uniform within a given panel. Furthermore, the skin thickness and the stiffener thickness are optimized concurrently (as was also done above), bringing the total number of design variables per panel to three. This variable quantity is several orders of magnitude less than the number of design variables per panel used in the topology optimization.

There are perhaps more accurate methods to model full-depth blade stiffeners than that shown in Fig. 13, but this method is used here because it presents a fair comparison with the topology optimization. In other words, the method of Fig. 13 is a subset of the topology design space, and could potentially be selected by the topology optimizer as a solution to Eq. 3 (though this is highly unlikely).

Convergence characteristics are shown in Fig. 14 for the topologically-optimized case (repeated from Fig. 5), and the case with solid full-depth stiffeners. Both cases utilize 10 inner-loop panel iterations per single outer-loop wing iteration. As expected, the full-depth stiffener case is heavier than the optimized topology case, by $7.43 \%$, directly due to the substantial imbalance in the size of the two design spaces. It can also be seen that the full-depth stiffener case experiences a stronger convergence of the wingbox mass objective function. The weak convergence of the optimized topology case was speculated above to be due to the discrepancy in computational cost needed to converge the macro-level sizing details and the local topological details. Obviously, no such discrepancy exists for the simpler case with full-depth stiffeners. 


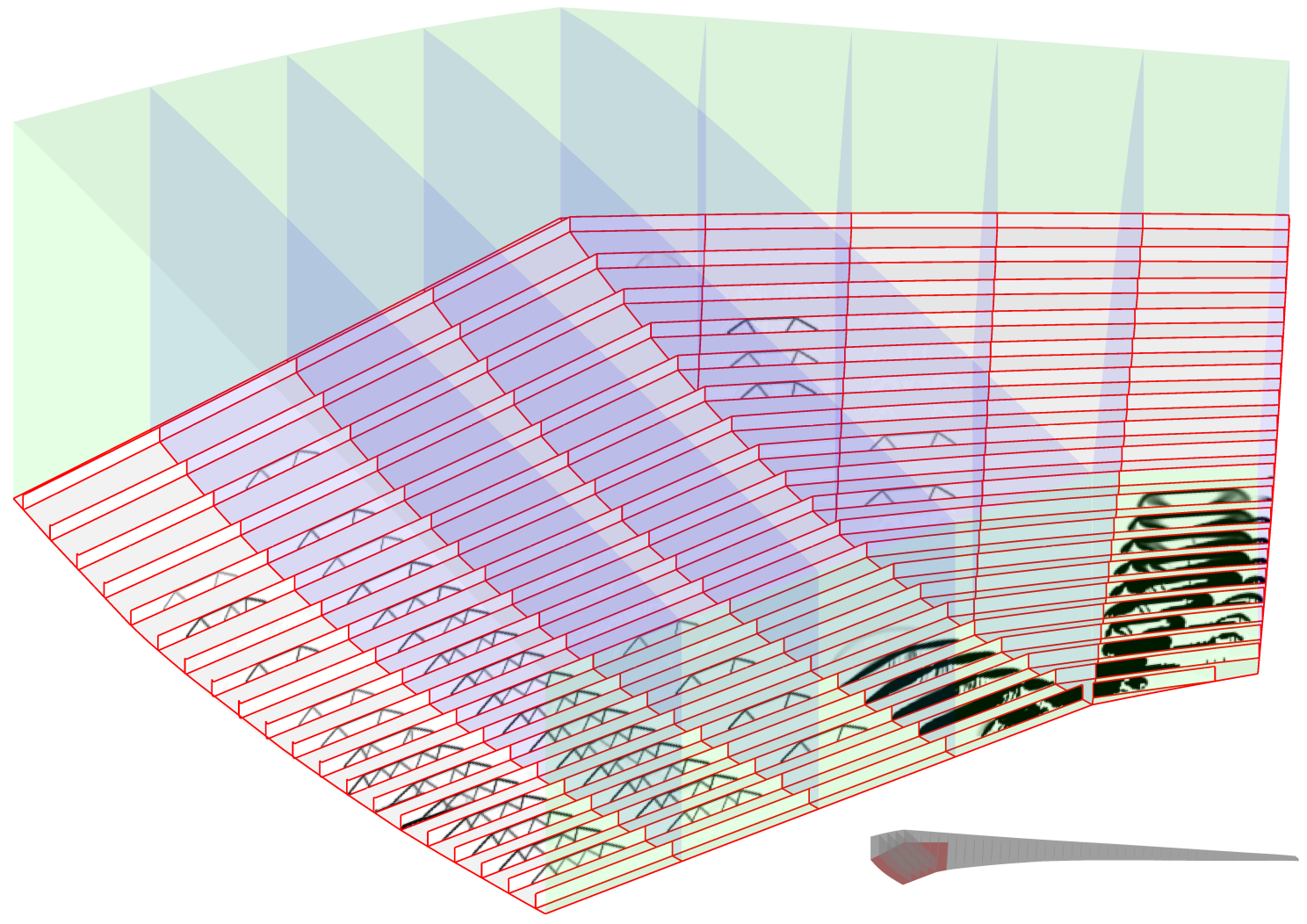

Figure 9. Lower skin topology near wing root.

Finally, panel and stiffener mass trends along the span are shown in Fig. 15, with the expected mass penalty for the case with solid full-depth stiffeners. The mass of the associated skin panels is typically higher as well, except in the lower skins near the root, where the topologically-optimized case is slightly heavier. Another interesting observation from Fig. 15 is seen in the stiffener mass at the lower skin near the root, where some full-depth stiffeners are substantially lighter than their topologically-optimized counterparts. This result is counterintuitive, and suggests that the topology optimizer struggles to locate reasonable designs in these panels (whereas the far-simpler full-depth case, paradoxically, does not). Indeed the stiffener structures seen in this location in Fig. 9 are topological outliers compared to their adjacent counterparts, particularly at the trailing edge break geometry, where the local stresses are expected to be high.

\section{Conclusions}

This paper has demonstrated an aeroelastic topology optimization process, where a transport wingbox model (the Common Research Model) is outfitted with equally-spaced skin stiffeners, and a topology optimizer then introduces holes within the webs to lighten those members. In order to facilitate rich localized topological features in a large wing model, a global-local approach is adopted, wherein aeroelastic loads are computed at the global wing-level, but topology optimization takes place within a local panel-level. Each panel is optimized concurrently, to minimize mass under stress, buckling, and various adjacency constraints. The panels are not driven to complete convergence, but instead partially optimized, after which the wing is reassembled, aeroelastic loads are recomputed, and the process repeats.

The final process results in a set of largely (but not entirely) well-resolved topologies across the wingbox. In order to baseline the optimal topology, the optimization is rerun with the restriction that all stiffener topologies may only resemble full-depth stiffeners without lightening holes. The final mass of this latter case is found to be $7.43 \%$ heavier than the topologically-optimized case. This weight change is substantial, though of course the manufacturing cost of the optimal topology case is much higher than the simpler case with solid full-depth stiffeners. 


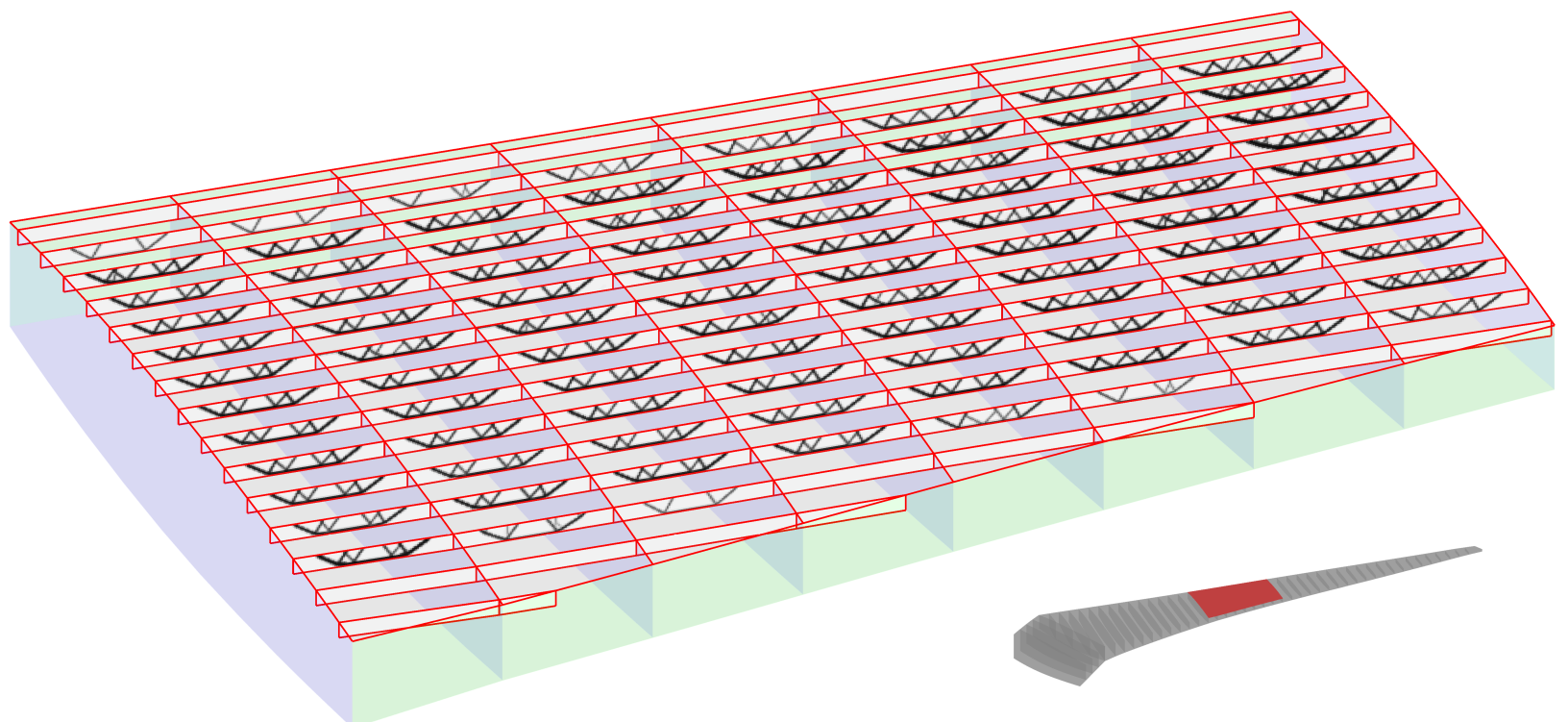

Figure 10. Upper skin topology near midspan.

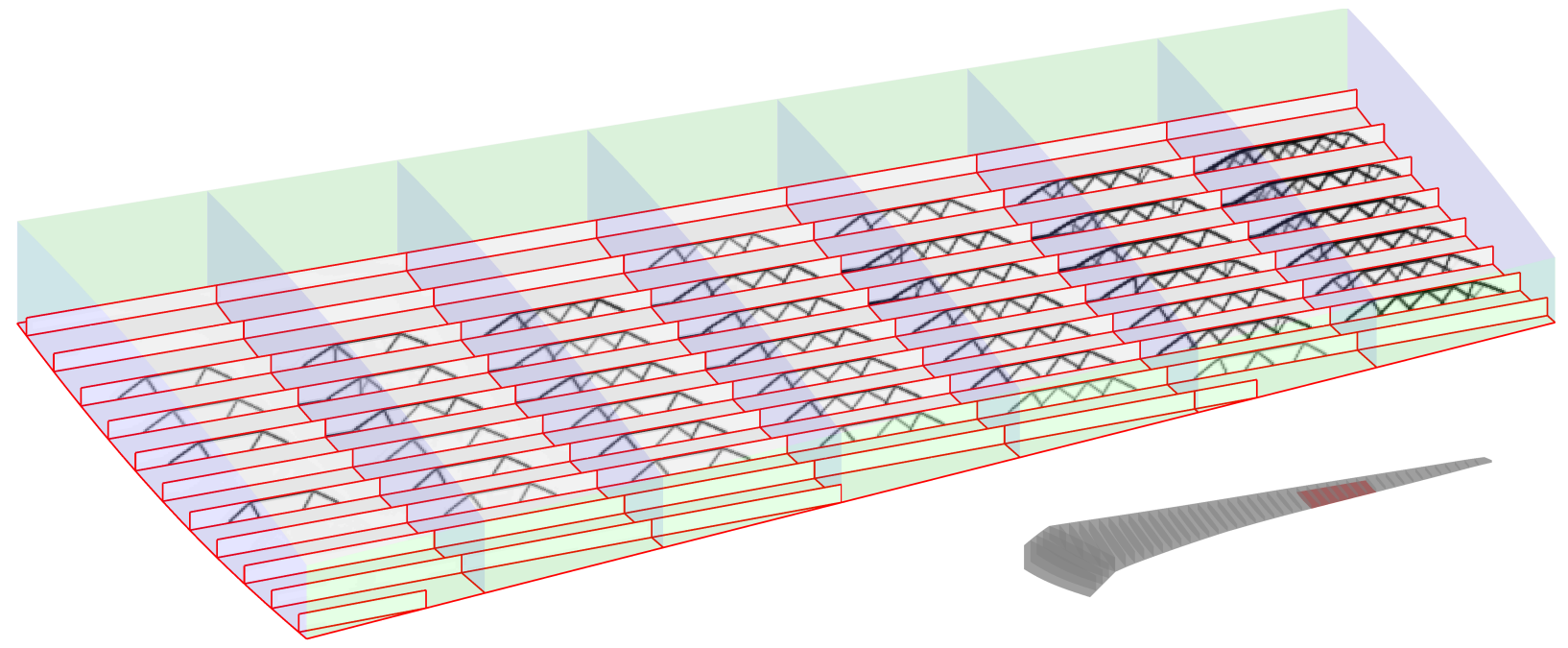

Figure 11. Lower skin topology near midspan.

There are a number of interesting extensions to this effort that may be considered as future work. Most obviously, it is of interest to also optimize the topologies within the rib webs, ${ }^{3,5}$ a process presumably driven by crushing loads ${ }^{8}$ among other concerns. As ribs become weaker, the assumption made here that buckling mechanisms are confined to a single rib bay panel becomes less-valid (overly-conservative), potentially requiring a global (wing-level) eigenvalue model. Geometric nonlinearities may also be of interest: in addition to being a key driver for rib crushing loads, they can also provide post-buckling stiffness, rendering the linear buckling failure envelope too conservative. Finally, various design considerations (aeroelastic flutter, for example) can only be accounted for at the wing-level, and have no local panel-level basis. To accommodate such metrics, the tools used here would have to include optimization at the global-level and the local-level (as opposed to the current work, where optimization is only conducted at the local panel-level). 


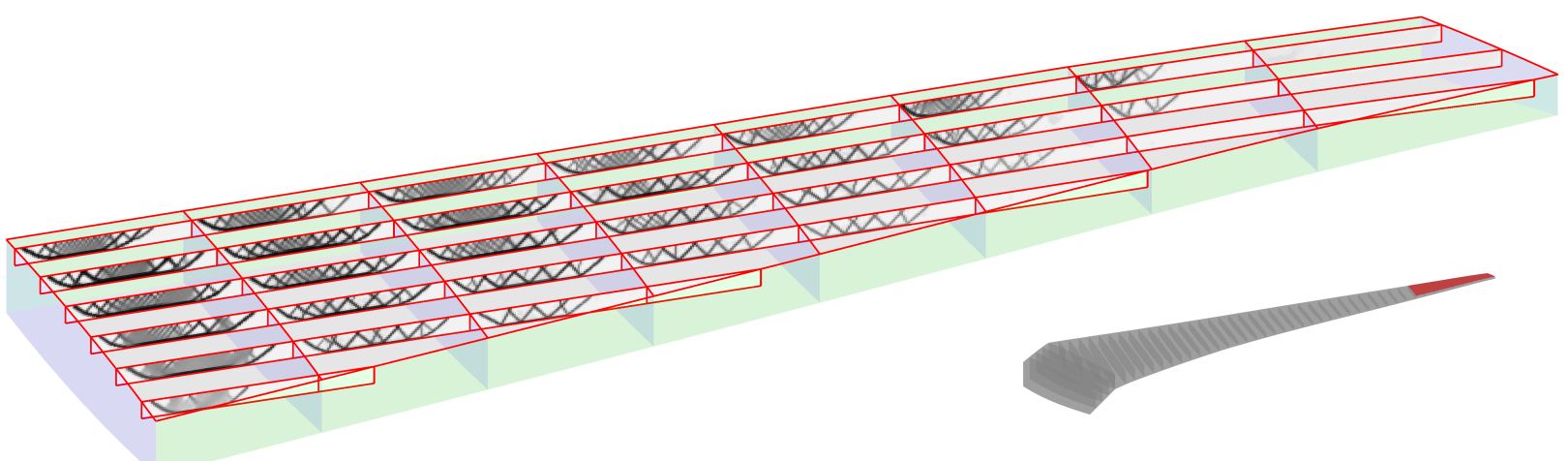

Figure 12. Upper skin topology at wing tip.

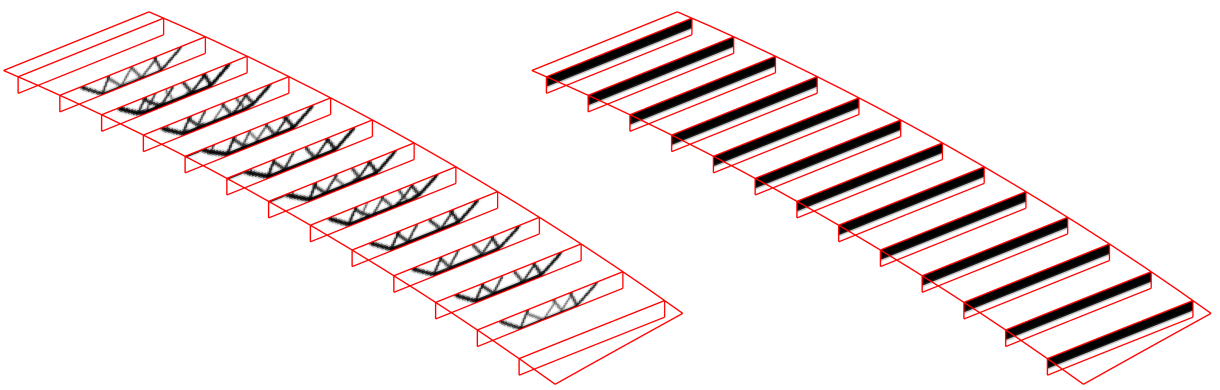

Figure 13. Sample panel with topologically-optimized stiffeners (left) versus solid full-depth stiffeners (right).

\section{Acknowledgments}

This work is funded by the NASA Advanced Air Transport Technologies project.

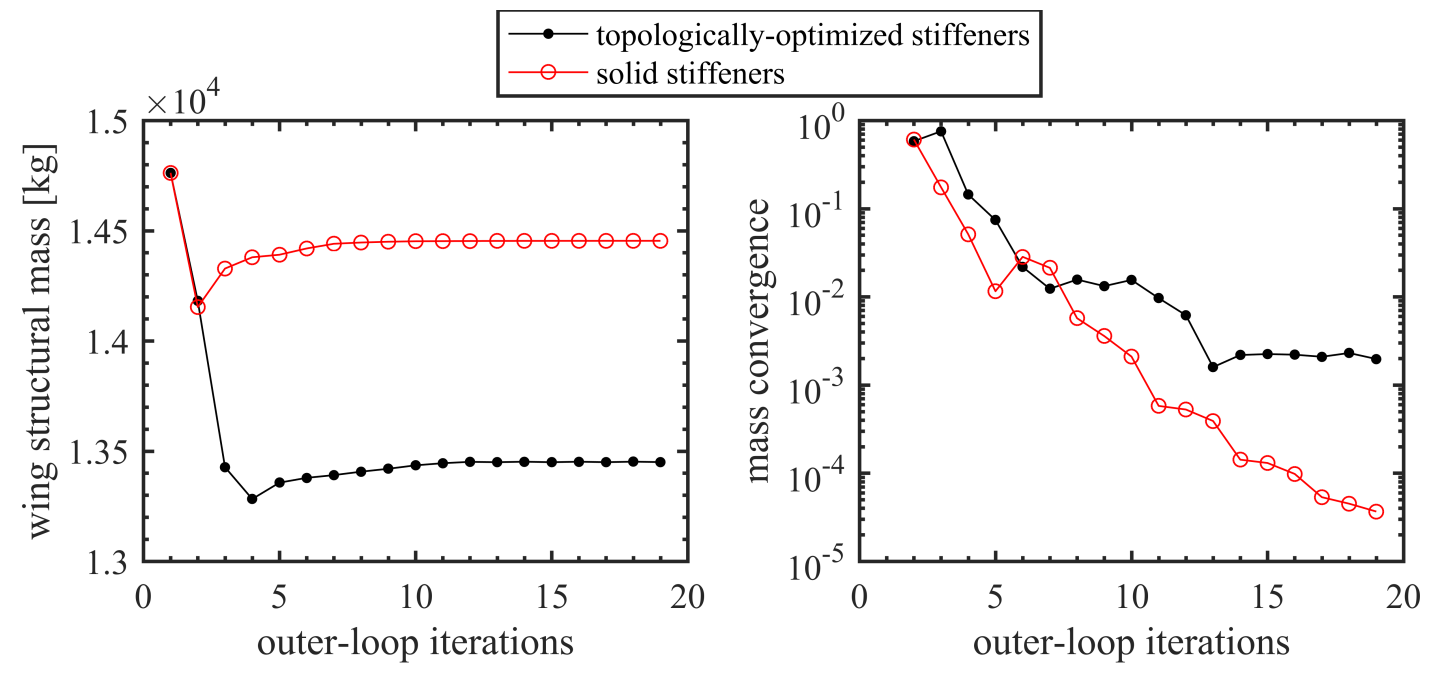

Figure 14. Outer-loop wing-level convergence histories, for the topology optimization case and the case with full-depth stiffeners. 

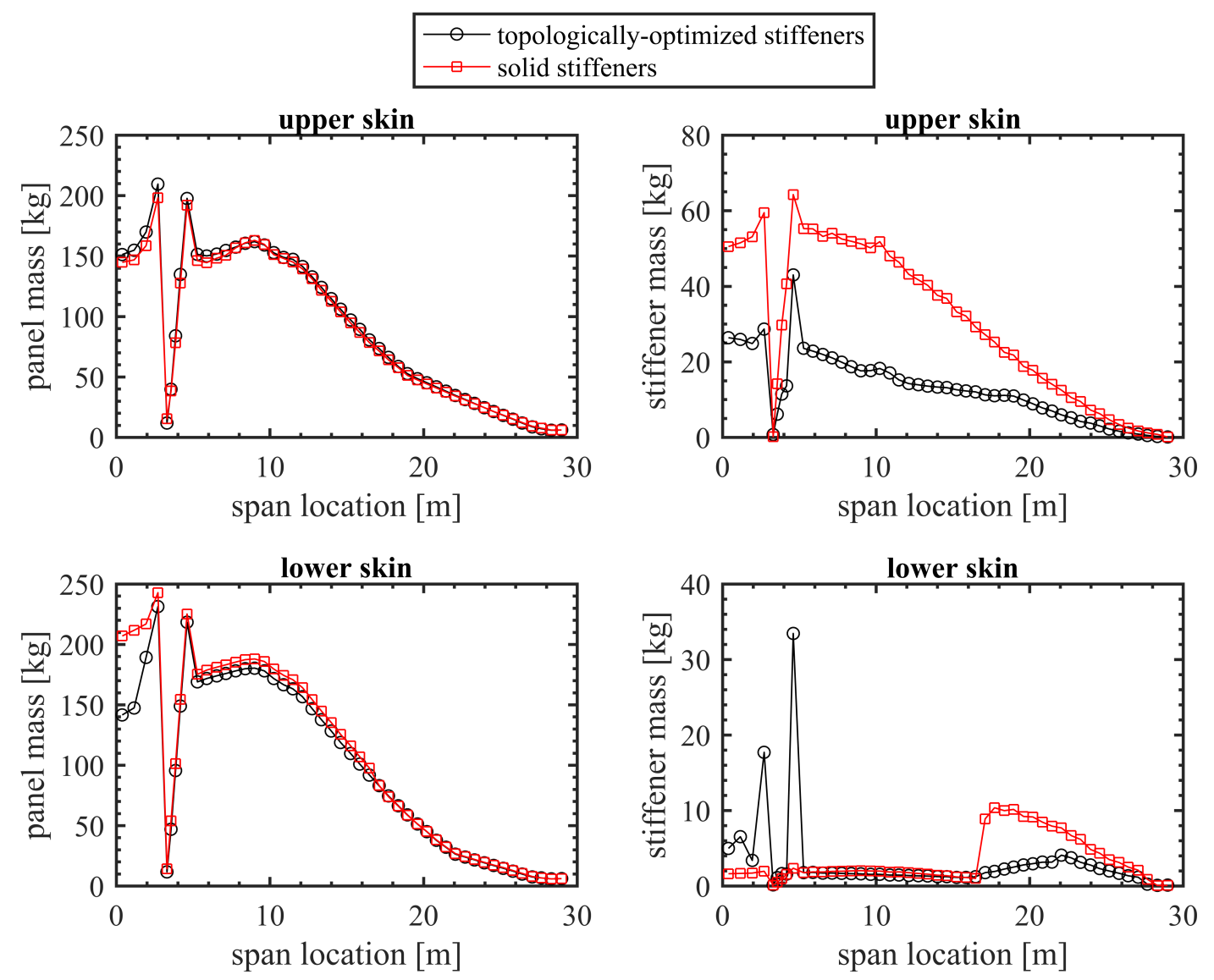

Figure 15. Skin panel and stiffener mass distributions.

\section{References}

\footnotetext{
${ }^{1}$ Locatelli, D., Mulani, S., Kapania, R., "Wing-Box Weight Optimization Using Curvilinear Spars and Ribs (SpaRibs)," Journal of Aircraft, Vol. 48, No. 5, pp. 1671-1684, 2011.

${ }^{2}$ Kolonay, R., Kobayashi, M., "Optimization of Aircraft Lifting Surfaces using a Cellular Division Method," Journal of Aircraft, Vol. 52, No. 6, pp. 2051-1684, 2015.

${ }^{3}$ Maute, K., Allen, M., "Conceptual Design of Aeroelastic Structures by Topology Optimization," Structural and Multidisciplinary Optimization, Vol. 27, No. 1, pp. 27-42, 2004.

${ }^{4}$ Stanford, B., Beran, B., Bhatia, M., "Conceptual Design of Aeroelastic Structures by Topology Optimization," Journal of Aircraft, Vol. 51, No. 3, pp. 938-944, 2014.

${ }^{5}$ Stanford, B., Dunning., P. "Optimal Topology of Aircraft Rib and Spar Structures under Aeroelastic Loads," Journal of Aircraft, Vol. 52, No. 4, pp. 1298-1311, 2015.

${ }^{6}$ James, K., Kennedy, G., Martins, J., "Concurrent Aerostructural Topology Optimization of a Wing Box," Computers and Structures, Vol. 134, No. 1, pp. 1-17, 2014.

${ }^{7}$ Dunning, P., Stanford, B., Kim, H., "Coupled Aerostructural Topology Optimization using a Level Set Method for 3D Aircraft Wings," Structural and Multidisciplinary Optimization, Vol. 51, No. 5, pp. 1113-1132, 2015.

${ }^{8} \mathrm{Niu}, \mathrm{M} .$, Airframe Structural Design, Conmilit Press Ltd., Hong Kong, 1988.

${ }^{9}$ Eldred, M., Venkayya, V., Anderson, W., "Mode Tracking Issues in Structural Optimization," AIAA Journal, Vol. 33, No. 10, pp. 1926-1933, 1995.

${ }^{10}$ Stroud, W., Agranoff, N., "Minimum-Mass Design of Filamentary Composite Panels under Combined Loads: Design Procedure Based on Simplified Buckling Equations," NASA TN D-8257, 1976.

${ }^{11}$ Schmidt, L., Mehrinfar, M., "Multilevel Optimum Design of Structures with Fiber-Composite Stiffened Panel Components," AIAA Journal, Vol. 20, No. 1, pp. 138-147, 1982.

${ }^{12}$ Ragon, S., Gürdal, Z., Haftka, H., Tzong, T., "Bilevel Design of a Wing Structure Using Response Surfaces," Journal of Aircraft, Vol. 40, No. 5, pp. 985-992, 2003.

${ }^{13}$ Ciampa, P., Nagel, B., Tooren, M., "Global Local Structural Optimization of Transportation Aircraft Wings," AIAA Structures, Structural Dynamics, and Materials Conference, Orlando, FL, April 12-15, 2010.

${ }^{14}$ Vassberg, J., DeHaan, M., Rivers, S., Wahls, R., "Development of a Common Research Model for Applied CFD Validation Studies," AIAA Applied Aerodynamics Conference, Honolulu, Hawaii, August 10-13, 2008.
} 
${ }^{15}$ Kenway, G., Martins, J., Kennedy, G., "Aerostructural Optimization of the Common Research Model Configuration," AIAA Aviation Conference, Atlanta, GA, June 16-20, 2014.

${ }^{16}$ Bendsøe, M., Sigmund, O., Topology Optimization - Theory, Methods, and Applications, Springer, New York, 2003.

${ }^{17}$ Kreisselmeier, G., Steinhauser, R., "Systematic Control Design by Optimizing a Vector Performance Index," International Federation of Active Controls Symposium on Computer-Aided Design of Control Systems, Zurich, Switzerland, 1979.

${ }^{18}$ Svanberg, K., "The Method of Moving Asymptotes - a New Method for Structural Optimization," International Journal for Numerical Methods in Engineering, Vol. 24, No. 2, pp. 359-373, 1987.

${ }^{19}$ Ragon, S., Gürdal, Z., Starnes, J., "Optimization of Composite Box-Beam Structures Including Effects of Subcomponent Interactions," NASA CR 199667, 1995.

${ }^{20}$ Wang, M., Wang, X., Guo, D., "A Level Set Method for Structural Topology Optimization," Computer Methods in Applied Mechanics and Engineering, Vol. 192, No. 1, pp. 227-246, 2003. 